\title{
Correction to: Body centered non-fullerene acceptors substitution on triangular shaped Sub-phthalocyanines (SubPcs) based A-D-A organic solar cells: A step toward new strategies for better performances
}

\author{
Faheem Abbas $^{1,2}$. Usman Ali ${ }^{2,3,4}$ (D) Hafiz Muhammad Rizwan Ahmad ${ }^{5,6}$. \\ Aqsa Tallat ${ }^{2} \cdot$ Aamir Shehzad $^{7} \cdot$ Zonish Zeb $^{1} \cdot$ Iqbal Hussain $^{4,8} \cdot$ Aamir Saeed $^{4,8}$ \\ Published online: 27 December 2021 \\ (c) Springer Science+Business Media, LLC, part of Springer Nature 2021
}

\section{Correction to: Optical and Quantum Electronics (2022) 54:21 https://doi.org/10.1007/s11082-021-03413-w}

In the original publication of the article, the authors noticed errors in abstract, figure caption, reference, layout of tables and figures. These errors have been corrected with this Correction.

In Abstract, the first sentence should begin with the word "Four". The corrected sentence should read, "For Acceptor-Donor-Accepter (A-D-A) type of triangular shaped sub-phthalocyanines (SubPcs) donor molecules namely SubPcs-EDM (sub-phthalocyanines-ethylidene di-malononitrile as M1), SubPcs-ETFM (sub-phthalocyanines-ethylidene tetrafluoromalononitrile as M2), SubPcs-ETFOM (sub-phthalocyanines-ethylidene tetrafluoro-oxo

The original article can be found online at https://doi.org/10.1007/s11082-021-03413-w.

Usman Ali

usmanqureshi@iccas.ac.cn; usmanqureshi320@gmail.com

1 Key Lab of Organic Optoelectronics and Molecular Engineering of Ministry of Education, Department of Chemistry, Tsinghua University, Beijing 100084, People's Republic of China

2 Department of Chemistry, University of Agriculture, Faisalabad 38040, Pakistan

3 Institute of Chemistry, Beijing National Laboratories for Molecular Sciences, Key Laboratories of Organic Solids, Chinese Academy of Sciences, Beijing 100190, People's Republic of China

4 University of Chinese Academy of Sciences, Beijing 100190, People's Republic of China

5 Center for Advancing Materials Performance From the Nanoscale, State Key Laboratory for Mechanical Behavior of Materials, Xi' an Jiaotong University, Xi' an 710049, People's Republic of China

6 Department of Physics, International Islamic University, Islamabad 44000, Pakistan

7 State Key Laboratory for Structural Chemistry of Unstable and Stable Species, Institute of Chemistry, Chinese Academy of Sciences, Beijing 10090, People's Republic of China

8 Key Laboratory of Green Process and Engineering, Institute of Process Engineering, Beijing Key Laboratories of Ionic Liquids Clean Process, Chinese Academy of Sciences, Beijing 100190, People's Republic of China 
malononitrile as M3) and SubPcs-EOM (sub-phthalocyanines-ethylidene oxo malononitrile as M4) have been designed for computing its optoelectronic properties with state-of-the-art density functional theory B3LYP/LanL2DZ (d, p) model."

In the caption of Fig. 7, the symbol "=>" was incorrectly published. The corrected caption is given below:

"Fig. 7 Charge transfer mechanism between $\mathrm{S} 0=>\mathrm{S} 1$ state with exciton binding energy"

The reference, "Cui, Y., Yao, H., Hong, L., Zhang, T., Tang, Y., Lin, B., Bi, P. (2019). 17\% efficiency organic photovoltaic cell with superior processability. Natl. Sci. Rev (http://creat ivecommons.org/licenses/by/4.0/)" has been removed.

The layout for Tables 1 and 3 and Figs. 5, 6 and 7 have been corrected. The corrected tables and figures are presented below:

The original article has been corrected.

Table 1 HOMO, LUMO, energy gap (HOMO-LUMO), first singlet $\left(\mathrm{S}_{1}\right)$ state excitation energy $\left(\mathrm{E}_{\text {opt }}\right)$ and binding energy of exciton at $\mathrm{S}_{1}$ excited state with B3LYP and LanL2DZ basis set are given in the unit of electron volt (eV)

\begin{tabular}{llllll}
\hline Molecules & $\mathrm{E}_{\text {Hомо }}$ & $\mathrm{E}_{\mathrm{LUMO}}$ & $E_{\mathrm{H}-\mathrm{L}}$ & $E_{\text {opt }}$ & $E_{\mathrm{b}}$ \\
\hline $\mathrm{R}$ & -5.93 & -3.22 & 2.71 & 2.385 & 3.323 \\
M1 & -6.15 & -3.59 & 2.56 & 2.071 & 2.183 \\
M2 & -6.13 & -3.64 & 2.49 & 1.982 & 2.444 \\
M3 & -6.25 & -3.77 & 2.48 & 1.921 & 2.308 \\
M4 & -6.11 & -3.42 & 2.69 & 2.220 & 2.572 \\
\hline
\end{tabular}

Table 3 Reorganization energy of hole and electron in the unit of electron volt (eV), ground state, excited state and the difference of dipole moment between excited and ground states in the unit of Debye are shown below

\begin{tabular}{llllll}
\hline Molecules & $\lambda_{h}$ & $\lambda_{e}$ & $\mu_{g}$ & $\mu_{e}$ & $\mu_{e-} \mu_{g}$ \\
\hline $\mathrm{R}$ & 0.063 & 0.282 & 2.073 & 2.324 & 0.251 \\
M1 & 0.061 & 0.315 & 7.286 & 8.113 & 0.827 \\
M2 & 0.054 & 0.102 & 8.056 & 8.844 & 0.788 \\
M3 & 0.056 & 0.382 & 7.136 & 7.534 & 0.398 \\
M4 & 0.053 & 0.611 & 5.715 & 6.292 & 0.577 \\
\hline
\end{tabular}




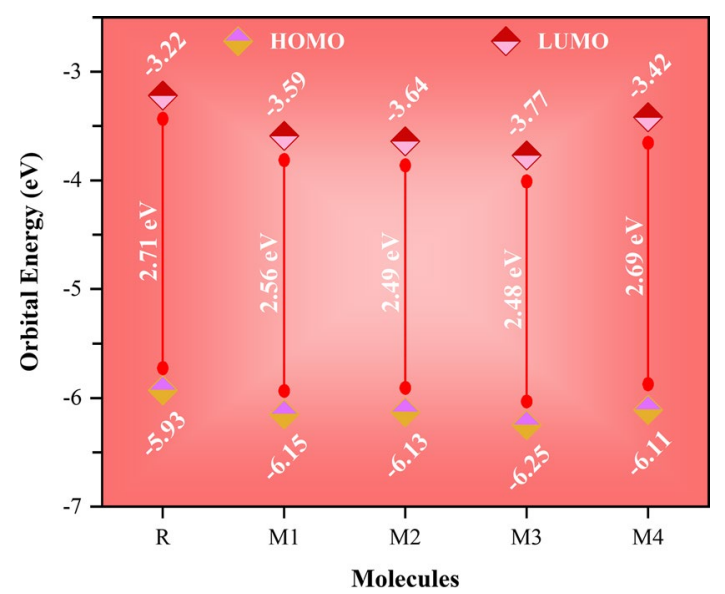

Fig. 5 Energy gap difference between HOMO to LUMO energy orbitals $(\mathrm{S} 0=>\mathrm{S} 1)$

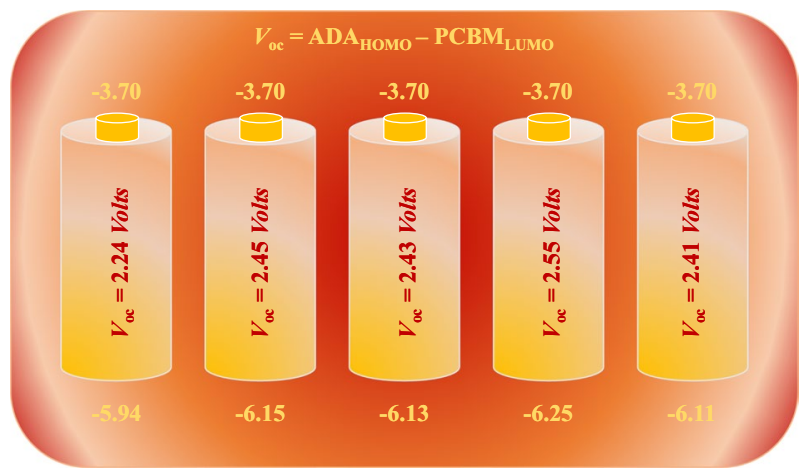

Fig. 6 Open circuit voltages of designed molecules with reference to PCBM LUMO

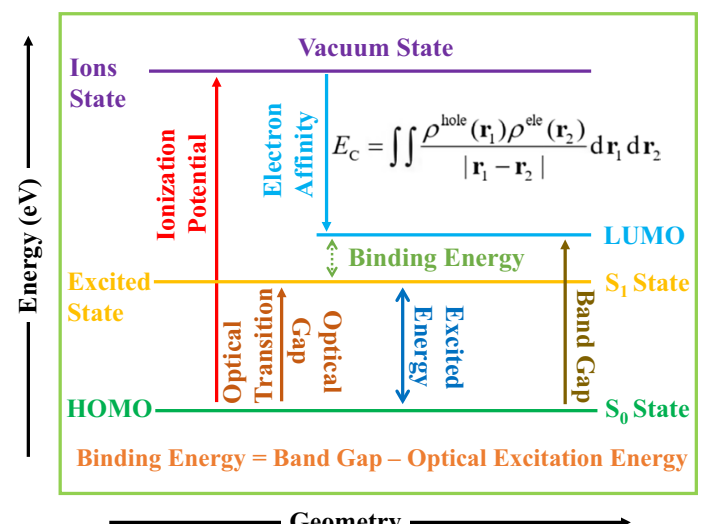

Fig. 7 Charge transfer mechanism between $\mathrm{S} 0=>\mathrm{S} 1$ state with exciton binding energy

Publisher's Note Springer Nature remains neutral with regard to jurisdictional claims in published maps and institutional affiliations. 